\title{
Research of Training Airspace Planning based on Genetic Algorithm
}

\author{
Jiacheng Ma, Dengkai Yao, and Guhao Zhao \\ Air Traffic Control College, Air Force Engineering University, Xi'an 710051, China \\ 727032612@qq.com
}

\begin{abstract}
Airspace planning of tactical training is a centralized planning, which is typical for Air Force tactical training. Because of the complexity of airspace and the diversity of training courses, artificial packing can't guarantee the utilization rate of airspace. Due to the irregularities of airspace, the minimum horizon merit-based insertion algorithm was proposed based on analysis of BL algorithm considering the reasonable utilization of surrounding airspace; On account of airspace limitation, selection operator, crossover operator and fitness function were established based on basic genetic algorithm, and for the purpose of packing optimization, genetic algorithm and improved packing algorithm were combined. The results show that the algorithm can ensure the utilization of airspace. The above method may provide a scientific basis for airspace planning of tactical training in real life.
\end{abstract}

Keywords: airspace; genetic algorithm; packing optimization; BL algorithm

\section{基于遗传算法的训练空域规划方法研究}

\author{
马嘉呈, 姚登凯, 赵顾影 \\ 空军工程大学空管领航学院, 西安, 中国
}

摘 要: 战术训练空域规划是对航空兵战术训练所需空域的集中规划。由于空域的复杂性和训 练科目的多样性, 人工排样无法保证空域的利用率。针对空域的不规则性, 在分析研究BL算 法的基础上, 提出了基于最低水平线的择优插入算法, 考虑周边空域的合理利用; 针对空域 的有限性, 在基本遗传算法的基础上加以改进, 编写了选择算子, 交叉算子及适应度函数, 并将遗传算法与改进后的排样算法结合来进行排样优化。结果表明该算法能够保证空域的利 用率。上述方法可为实际中的空域战术训练规划提供科学依据。

关键词: 空域; 遗传算法; 排样优化; BL算法

\section{1. 前言}

随着我空军军事训练转型的推进, 军事训练时间、战术训练比重不断提高, 多兵机种合练、 体系对抗训练已成常态, 对空域等资源需求逐年增加。同时, 我国民用航空运输进入持续快 速发展的新阶段, 对空域的需求也日益迫切。因此, 通过合理规划战术训练空域, 将空域需 求矛盾紧张的各机场区域内的战术训练飞行集中到战术训练空域实施, 既有利于充分发挥第 三代主战装备航程远、火力杀伤范围大的优势。同时, 又优化了空域结构, 有效缓解军民空 域需求的矛盾问题。

对于训练空域的规划, 目前国内没有相对成熟的计算方法和理论体系, 通常情况下, 主要是 依靠参谋人员根据人为经验划设, 这也使得空域的利用率和灵活性都比较差, 而利用计算机 来进行辅助设计则可以提高工作效率。此类问题类似于排样问题, 由于复杂度高, 无法完全 求解。目前, 国内外对于解决排样问题的算法较多, 解决这类问题的近似算法, 如禁忌搜索 ( tabu search) 、模拟退火（ simulated annealing）、遗传算法（genetic algorithms）等。 文献 [1] 将人的经验和启发式思想结合用于解决矩形件的排样问题, 根据其长宽, 按照左下原 则来进行排样; 文献 [2]研究了矩形件正交排样的遗传算法求解, 将个体的编码看成排列通过 
BL 算法来转化为排样图; 文献 [3]对 BL 算法进行了改进, 更好地解决了编码转化的问题; 文 献 [4] 将不规则件拟合为凸多边形, 再将凸多边形组合为正六边形, 然后对不规则件求解排样 问题。

相较于文献 [1-4] 中的排样问题, 解决战术训练空域规划问题时有其自身的独特性。其一是需 要解决训练区域的不规则性, 通常情况下, 机场所辖的训练区域是不规则形的, 这无疑增加 了问题的复杂性。而参考文献 [1-3]中的遗传算法均是针对规则的矩形区域来进行排样的, 参 考文献 [4] 中是解决的是不规则零件在规则区域内的排样问题。其二是需要考虑训练区域的有 限性, 各个训练科目的空域不得超出指定空域, 否则会带来不必要的麻烦。而且并不是所有 的训练科目均可排入。这也是其它排样问题的不同之处。本文利用一种新的解码方法来解决 不规则区的排样问题, 并重新定义一种新的适应度函数作为新的评判标准, 采用一个近似算 法即遗传算法来寻求最优解，以此来解决有限空域内的训练科目规划问题。

\section{2. 战术训练空域规划模型}

在对战术训练空域规划的过程中需要对实际中考虑的因素进行适当的简化, 并通过恰当的方 式来表达, 因此本节中主要是对训练空域规划模型中的影响因素以及其几何模型进行描述, 阐述规划过程中的一些前提条件, 为之后的排样算法做铺垫。

2.1 战术训练空域的影响因素

在实际的航空兵战术训练过程中, 由于受到多种因素的制约, 各个科目所需的训练空域大小 不尽相同, 这也给空域规划带来了相应的难度。而其中主要的影响因素分为以下几个方面:

1) 武器装备性能

战术训练空域的大小必须以满足武器装备数量各性能要求为最低标准。空域的长度与宽度必 须满足作战飞机从机动状态改平飞后至少能够完成一个完整的搜索、发现过程。同时, 随着 装备的增多和性能的提升, 所需的空域范围也不同。

2) 训练内容设置

不同的战术训练课目，对空域也提出了不同的要求。如合同战术对抗训练等科目，由于参训 兵力的增加, 作战范围广, 使所需空域范围也增大; 而其它课目如格斗课等对空域的需求要 小得多，划设的不同战术训练空域必须满足该科目的最低标准。

3）当地空域资源情况

主要包括天气因素 (如: 年均可飞天气) 、周围机场分布、军民航线、所在区域地形地貌以 及空中禁飞区等客观条件, 这些是在进行训练空域规划时必须考虑的因素。

\section{4) 其他因素}

主要包括当地航空管制能力和各种保障设施的保障能力。如我国局部地区仍采用非雷达管制 方式进行航空管制, 对空中多批次、多目标管制能力较弱, 则不适宜建设大型战术对抗训练 空域, 而保障能力不足也会对训练飞行造成负面影响，甚至危及飞行安全。

综上所述, 战术训练空域的大小并不相同, 这也增加了个体的多样性; 整个训练空域由于受 到各因素的限制, 通常是不规则的。同时考虑到空域的现状, 训练空域的容纳能力是有限的, 并不所有的训练科目都能被容纳。这此都给空域的规划增加了一定的难度。

\section{2 模型建立}

战术训练空域的规划问题是指在该机场所辖的训练区域内分配多个大小不同的训练空域, 使 得所辖训练区的利用率最大, 其分配需要满足以下条件：（1）不同训练科目所占空域之间不 能重叠 (2) 训练科目所占空域需分配在该机场所辖的训练区域内。本文讨论的整个训练空域 是凸多边形的, 而各个训练科目则抽象为矩形块结构。

整个凸多边形的训练空域, 可以用其顶点坐标按照一定顺序来进行描述, 空域边界 Loop 可用 $\mathrm{Loop}=\left\{\mathrm{P}_{1}, \mathrm{P}_{2}, \mathrm{P}_{3}, \mathrm{P}_{4}, \mathrm{P}_{5}\right\}$ 来表示。如图 1 所示。 


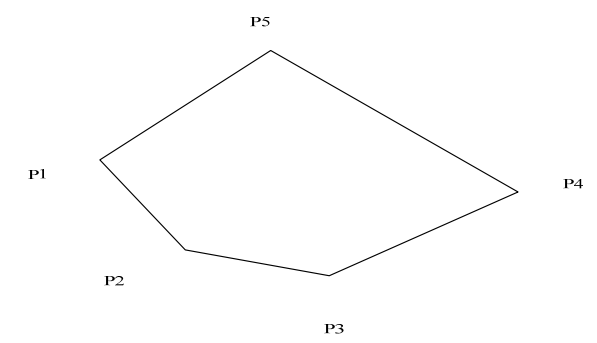

图 1 空域边界示意图

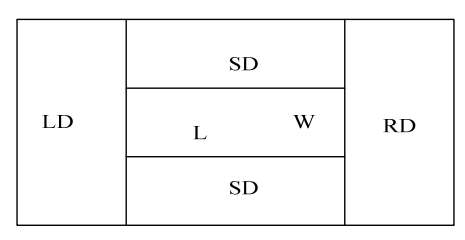

图 2 各科目所需空域示意图

各个训练空域为矩形结构, 除科目本身所需的空域外, 还要考虑相应的安全间隔。即 $\mathrm{B}=\{\mathrm{L}, \mathrm{B}, \mathrm{LD}, \mathrm{SD}, \mathrm{RD}\}$, 如上图 2 所示。

训练空域规划就是按照一定的规则, 在满足约束条件的情况下寻求空域利用率最佳的规划方 案。矩形块的各个顶点不得超出空域边界。如式 1 所示, 其坐标 $\left(X_{i j}, Y_{i j}\right)$ 应满足的条件为 $\left(X_{i j}, Y_{i j}\right) \in \prod($ Loop $)$

其中, $\prod$ (Loop) 为整个空域的外围边界。

\section{3. 改进遗传算法描述}

针对训练空域的不规则性, 多样性和有限性, 本文先对整个训练空域进行预处理, 再将各个 战术训练空域编码, 在 BL 算法的基础上作出相应的改进, 寻求新的解码方式。根据战术训练 空域规划的特点编写相应的适应度函数。

3.1训练空域的预处理

由于空域的不规则性, 首先要对整个的训练空域进行离散化处理, 具体步骤如下:

1) 将整个训练空域以图像的形式存储在内存当中

2) 将图像转化为位图 (0/1 形式) 进行存储

3) 人为划分区间, 并取置信度为 0.75 , 将位图转化为矩阵 $V$ 。

通过上述操作, 将各个训练空域转化为矩阵的形式存储, 以便进行后续的操作。

3. 2 可行解的编码方法

对于有 $\mathrm{n}$ 个训练任务的空域划设问题, 首先用 $1^{\sim} \mathrm{n}$ 依次对每个训练所需的空域进行编号, 则 问题最终的目标解可表示为数字串的形式, 即 $\mathrm{P}=\left\{\mathrm{P}_{1}, \mathrm{P}_{2}, \cdots, \mathrm{P}_{\mathrm{n}}\right\}$ 。其中, $\mathrm{n}$ 表示总的训练任务, $\mathrm{Pi}$ 是整数 $\left(1 \leqslant\left|P_{i}\right| \leqslant n\right)$, 有正负之分, 当 $P$ 为负值的时候, 表示该任务对应的空域先旋转 90 度再参与排放（默认的训练空域宽大于高）

采用这种数字串形式的好处在于可以更加便捷地改变排列方案, 并且每个方案都有唯一的序 列与之对应。每个序列都代表一个基因组, 之后的交叉和变异算子操作就是对数字串操作, 从而产生新的解

\section{3 可行解的解码方法}

对可行解进行编码后, 快速地求出其相应的排样图是关键所在。利用此排样图可进一步求出 空域的利用率。文献 [1] 研究了矩形正交排样的遗传算法求解, 其基本思想是将个体的编码视 作一个排列, 通过 BL (Bottom Left) 算法将编码转化为相应的排样图。但其是针对一个规 则区域进行排样, 而整个训练空域是不规则而且有限的, 显然此算法是不太适应的。因此, 本文在 BL 算法的基础上提出了一种新的解码方法。

对于给定的不规则空域的排放问题, 本文采用的策略是: 针对每个数字串, 向后不断搜索可 以放下的矩形训练空域，直至在给定不规则空域内无法分配为止。具体步骤如下:

1) 将不规则区域作离散化处理, 并设置最高轮廓线和最低水平线为不规则区域的下边界

2) 对于训练区域 Pi 的排放, 先在最高轮廓线中选择高度最低的一段水平线作为最低水平线, 如有数段, 选择靠左的一段。若该水平线的宽度大于 Pi 的宽度, 则在该线段上靠左排放该区 域; 否则, 从 Pi 开始向后搜索可以放置的矩形区。 
3) 对于已经排放的训练区域 Pi, 需检测其顶点是否在给定的不规则区域内, 若是, 则确定该 训练区的最终位置, 并更新最高轮廓线; 反之, 寻找下一个适合的矩形训练区。若均无法放 置, 则查找与最低水平线高度相邻的水平线, 并将其作为新的最低水平线。

4) 重复上述两个步骤，直至在该不规则区域内找不到可以分配的训练区域为止。

5) 根据最后得到的排样矩阵生成排样图。如图 3 所示。

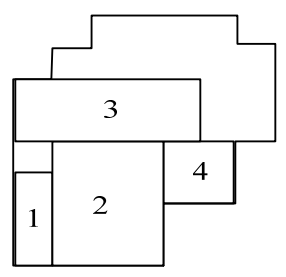

5

图 3 解码排放示意图

对于编码为 $\{-1,-2,3,4,5\}$ 的训练区排放, 在排入矩形 1,2 后, 最低水平线在最右侧, 当排 放 3 号训练区时, 由于此区域的宽度大于最低水平线的宽度, 故无法排放, 向后搜索可以排 放的矩形区, 将 4 号区域排入并提升最低水平线为最左边 1 号区域的上边界, 但由于其余训 练区宽度均大于此水平线, 因此无法排入，继续提升最低水平线为 2 号区域上边界，将 3 号 训练区排入并更新水平线为最右端剩余的线段，继续寻找可排入的训练区。因 5 号训练区的 顶点无法完全置入该区域内, 所以排放完毕。即在该不规则区域内, 只能放置 1, 2, 3, 4 号 训练区。

3.4 适应度函数

在遗传算法中通过适应度函数来评判每个个体的好坏。适应度值越大, 解的质量越好。在本 文中, 最大高度越低说明所需的空间越小。由于排样空有限, 排入的个数越多, 说明方案越 优。因此, 适应度函数的选取在考虑最大高度和可再利用空间两个因素的同时还要兼顾排入 的个数。如式 (2-3) 所示, 本文将适应度函数定义为:

$$
\begin{aligned}
& f(p)=n+\text { Aera } / \text { Aera0 } \\
& \text { Aera }=\sum S_{i}
\end{aligned}
$$

式中: $\mathrm{n}$ 代表排入的训练空域的数目; Aera 是排入的训练区域的总面积; Aera0 是最高轮廓 线以下的面积, 其比值的最高值是 1 。 Si 为各个训练区域的面积

\section{4. 遗传算法的求解过程}

遗传算法是一种借鉴生物界自然选择思想和自然遗传机制的全局随机搜索的算法。相较于罗 列法, 该方法在解决大空间全局寻优的问题时具有独特的优势。利用遗传算法可更加便捷地 寻找到利用率最大的规划方案。

4. 1 种群的初始化

随机生成 $\mathrm{n}$ 个 ( $\mathrm{n}$ 为偶数) 序列作为初始种群, 并利用下台阶算法求出种群中每个个体的适 应度, 将其按照从大到小的顺序排列。

\section{2 交叉操作}

将父辈群体中的个体随机两两配对, 进行交叉操作。为了更加真实地模拟自然界中的遗传特 征, 本文采用双亲配对交叉的方式来进行交叉操作。即：先将 $n$ 个序列两两配对生成 $n / 2$ 对 染色体, 我们假定 $L 1=\left\{1_{11}, 1_{12} \cdots 1_{1 n}\right\}$ 和 $L 2=\left\{1_{21}, 1_{22} \cdots 1_{2 n}\right\}$ 为已经配对的一对染色体。再随机选取 两个数字 $\mathrm{p}, \mathrm{q}(1 \leqslant \mathrm{p} \leqslant \mathrm{q} \leqslant \mathrm{n})$ 来进行交叉操作，具体步骤如下：在 $\mathrm{L} 1$ 上从第 $\mathrm{p}$ 个基因开始选取 $\mathrm{q}$ 个基因作为下一代染色体的前 $\mathrm{q}$ 个基因, 而剩余的 $\mathrm{n}-\mathrm{q}$ 个染色体按照 $\mathrm{L}_{2}$ 上出现的顺序接在 后面从而生成一个新的染色体。对 $1_{2}$ 进行相同的操作产生另一个新的染色体。当 $n=6, p=1, q=4$ 时, 如图 4 所示: 


\begin{tabular}{|c|c|c|c|c|c|c|}
\hline $\mathrm{L}_{1}$ & 5 & 6 & 4 & 3 & 2 & 1 \\
\hline $\mathrm{L}_{2}$ & 4 & 3 & 5 & 2 & 1 & 6 \\
\hline$L_{1}^{\prime}$ & 5 & 6 & 4 & 3 & 2 & 1 \\
\hline
\end{tabular}

图4 交叉操作图样

\section{3变异操作}

目前常见的变异方法有很多种，本文采用旋转变异的方法。具体操作如下：假定一个小概率 $\mathrm{Pm}$, 对每个基因都生成一个随机数 $\mathrm{P}$, 若 $\mathrm{P} \leqslant \mathrm{Pm}$ 则改变基因的正负, 即将基因所对应的训练 空域旋转 90 度来放置。还有一种变异为颠倒变异, 即随机选择 2 个变异位, 以一定的概率对 两个变异位之间的数颠倒顺序。当个数比较少的时候, 颠倒变异可以在一定程度上提高搜索 效率。但在此遗传算法中, 由于在解码过程中已经做出了动态的调整, 故在算法实现时, 只 考虑颠倒变异。

\section{4选择操作}

对于交叉和变异操作生成的 $\mathrm{n}$ 个新染色体依次求解其适应度函数值, 将其与父辈的染色体一 起根据适应度值的大小进行排列, 并选取前 $\mathrm{n}$ 个染色体来作为下一代的父本。不断重复上述 操作直到适应度函数值不再发生变化或者达到预定迭代次数为止, 来得到最终的最优解。

\section{5. 仿真分析}

为了测试该遗传算法对于有限不规则区域排样问题的有效性，进行下述的实例验证。

（一）某机场不同机型的不同训练科目所需的空域大小见表 1 。

表1各个训练区大小

\begin{tabular}{cccccc}
\hline 序号 & 长 & 宽 & 序号 & 长 & 宽 \\
\hline 1 & 10 & 8 & 2 & 4 & 4 \\
3 & 5 & 3 & 4 & 6 & 8 \\
5 & 8 & 8 & 6 & 10 & 5 \\
7 & 4 & 14 & 8 & 6 & 6 \\
9 & 7 & 5 & 10 & 4 & 14 \\
11 & 9 & 8 & 12 & 5 & 5 \\
13 & 13 & 10 & 16 & 7 & 8 \\
15 & 6 & 4 & & 7 & 7 \\
\hline
\end{tabular}

假定该机场所辖的训练区域如图 5 所示:
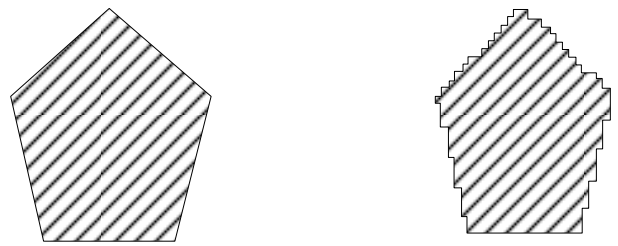

图 5 不规则训练空域图 图 6 离散化后的空域图

首先要对该不规则区域进行离散化处理, 并生成相应的矩阵。其离散化后的图形如 6 所示: 采用该遗传算法来进行计算, 设定种群规模为 $\mathrm{m}=20$, 迭代 100 次, 变异概率为 0.2 , 根据其 适应度函数来寻求最优解。通过计算得到的训练空域规划, 如图 7 所示, 。

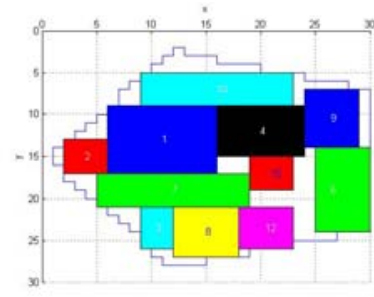

图 7 训练空域规划图

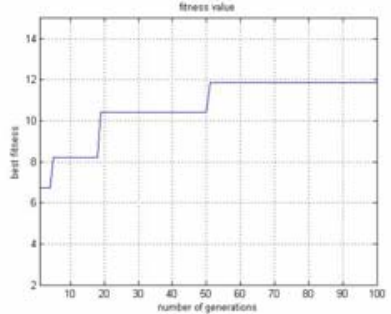

图 8 适应度函数值曲线图

此时整个规划图的适应度为 11.8796 。其求解过程中, 适应度函数值变化如图 8 所示: 
（二）假定该机场某天的训练科目只有三种，其大小分别为：（4，14）（8，8）（10，5）。 根据上述方法，同样可以得到其最优规划图，如下图 9 所示; 其最优适应度值为 7.7280。

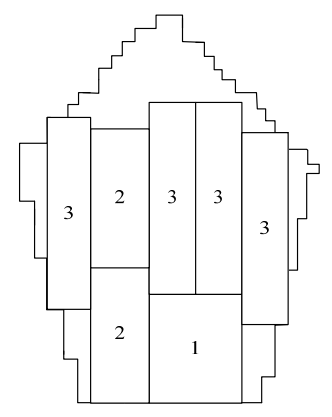

图 9 训练空域规划图

通过实例可以看出, 利用该算法可解决不规则空域规划问题。通过比较两个实例的利用率, 可发现当训练空域类型较多且有较小空域时，该算法的适用度会更高。在排样时间上，相较 于人为排样, 利用计算机来辅助排样大大缩短了排样时间, 尤其是排样种类较多时, 效果更 加显著。

\section{6. 结束语}

本文探讨了训练空域规划问题的遗传算法求解, 将其转化为有限不规则区域的排样问题, 并 在文献 [1] 中 BL 算法的基础上做了相应的改进, 并通过实例验证了其有效性。相比较于人为 划分训练区域，利用该算法可以提高空域规划的效率。本文在解码过程中所采用的搜索方式 和如何进一步提高求解的效率与精度, 还有待进一步的研究和改进

\section{致谢}

感谢国家空管科研课题基金：无人机空域运行安全关键技术研究（KGKT05140501）

\section{References}

[1]YNAASEE H H, ZNIOBER A S. Two-Dimensional Cutting Stock with Multiple Stock Sizes [J]. Journal of the Operational Research Society, 1991, 42(8):672

[2] Jakobs S. On genetic algorithms for the packing of polygons [J]. European Journal Operational Research, 1996, 88, (1): 165- 181

[3] Liu Dequan, TengHongfei. On genetic algorithm for the orthogonal packing of rectangles [J]. Mini-Micro Systems.1998,19(12):20 25

[4] Zhang Bao, Teng Hangfei, Shi Yanjun.Layout Optimization of Satellite Module Using Soft Computing Techniques. Applied Soft Computing Journal, 2008, 8(1):507-521.

[5] Andrea L, Silvano M, Michele M. Two-dimensional packing problem: a survey [J]. European Journal of Operation Research, 2002, 141: 241- 252. 